Revue des patrimoines

$4 \mid 2004$

Les réseaux de la villégiature

\title{
La Villégiature retrouvée : les réseaux de la recherche
}

\section{Claude Mignot}

\section{(2) OpenEdition}

1 Journals

Édition électronique

URL : http://journals.openedition.org/insitu/1289

DOI : 10.4000/insitu.1289

ISSN : 1630-7305

Éditeur

Ministère de la Culture

Référence électronique

Claude Mignot, "La Villégiature retrouvée : les réseaux de la recherche », In Situ [En ligne], 4| 2004, mis en ligne le 01 mars 2004, consulté le 21 septembre 2020. URL : http://journals.openedition.org/insitu/ 1289 ; DOI : https://doi.org/10.4000/insitu.1289

Ce document a été généré automatiquement le 21 septembre 2020.

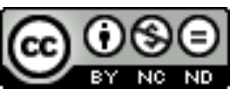

In Situ Revues des patrimoines est mis à disposition selon les termes de la licence Creative Commons Attribution - Pas d'Utilisation Commerciale - Pas de Modification 4.0 International. 


\title{
La Villégiature retrouvée : les réseaux de la recherche
}

\author{
Claude Mignot
}

\section{3-2003 : vingt ans après}

1 Il y a quelque trente ans, l'architecture du XIXe s. commençait à sortir de la polémique militante du modernisme pour entrer dans l'Histoire; les recherches sur l'architecture de la villégiature, elles, n'ont guère en France que vingt ans.

21974 : la défunte revue Monuments historiques de la France consacrait un premier numéro à la réévaluation de l'architecture du XIX ${ }^{\mathrm{e}}$ s. et Bruno Foucart, conseiller technique du nouveau ministre de la Culture, Michel Guy, établissait une première liste d'édifices à protéger ${ }^{1}$. On redécouvrait successivement l'Art nouveau (Horta, 1971), le néogothique (Le gothique retrouvé, 1979), l'éclectisme (Lille, le siècle de l'éclectisme, 1979 ; Davioud, 1981). En 1983 paraissaient, quasi simultanément, mon ouvrage, L'architecture au XIX ${ }^{e}$ siècle, et Le siècle de l'industrie par François Loyer, qui établissaient un premier bilan global de dix années de recherches, replacées dans un cadre international ${ }^{2}$.

3 Le phénomène de la villégiature n' y apparaissait encore que timidement: François Loyer s'intéressait au thème du casino et du Grand hôtel, mais il s'arrêtait plus sur les châteaux, les hôtels particuliers et l'habitat ouvrier que sur les villas de bord de mer; pour ma part, je montrais six villas balnéaires en France, en Angleterre, en Belgique et aux Etats-Unis, pour onze gratte-ciel ; tous deux, nous célébrions Paris, capitale du XIX ${ }^{\mathrm{e}}$ s., mais négligions les villes de villégiature, mesurant alors encore mal à quel point ces villes neuves, qui avaient occupé ce «territoire du vide» qu'était alors le rivage ${ }^{3}$, avaient été des laboratoires d'invention architecturale, typologique et stylistique, mais aussi d'urbanisme. 
Figure 1

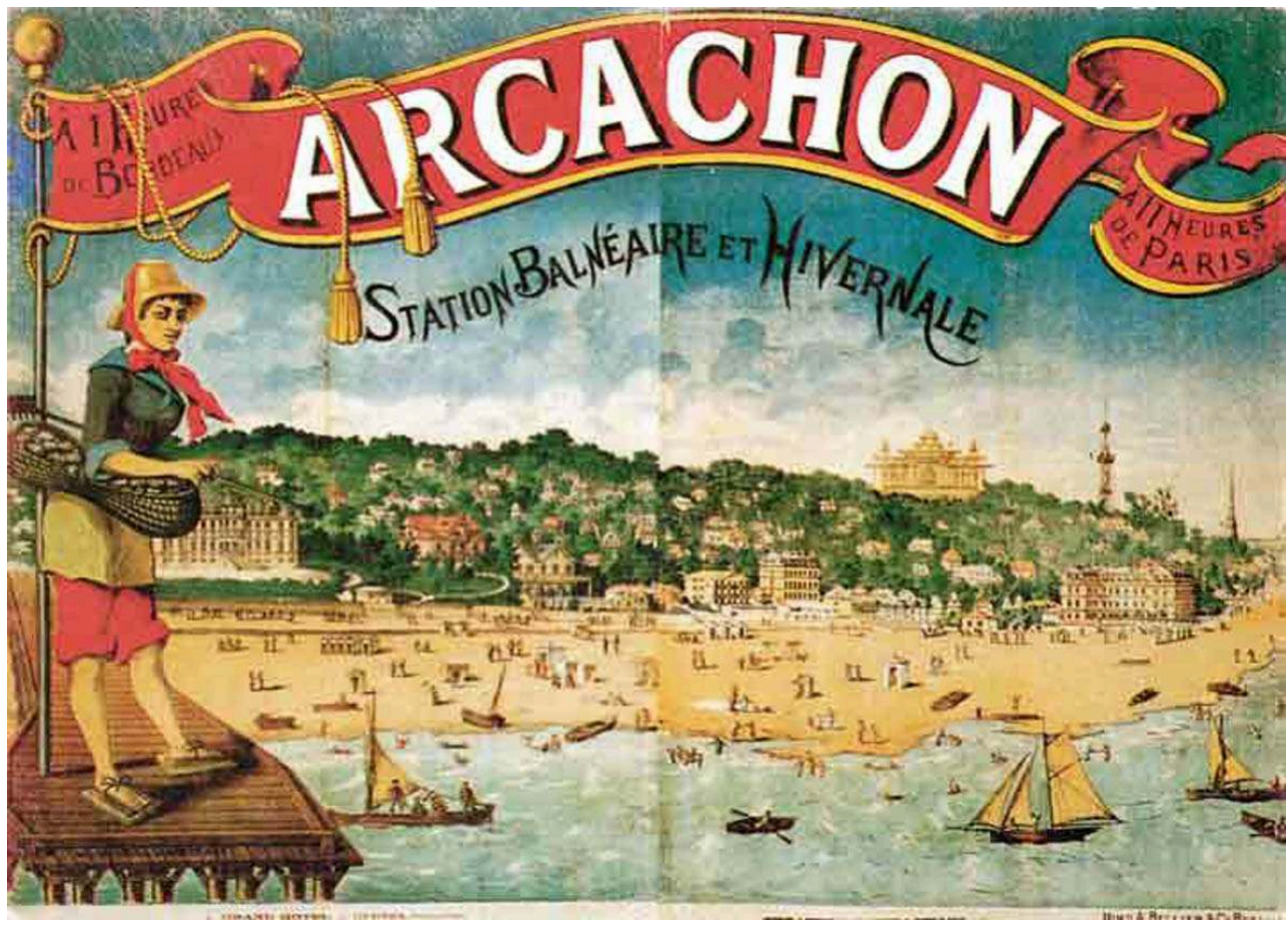

Arcachon (Gironde). Vue panoramique. Affiche éditée par la Société immobilière d'Arcachon vers 1880. Repro. Coll. B. Toulier. Extrait : Villes d'EAux. Paris, Dexia/Imprimerie Nationale, 2002, p. 31.

Les trois années 1982-1984 apparaissent rétrospectivement comme un grand tournant dans l'historiographie de l'architecture de la villégiature, dont l'importance commençait à être pressentie ${ }^{4}$. Sous l'impulsion de Maurice Culot, qui dirigeait le Département «Archives, histoire et documentation» de l'Institut français d'architecture (I.F.A.), paraissaient en 1982 et 1983 les premières monographies d'une station thermale, Vittel, et d'une station climatique, la ville d'hiver d'Arcachon ${ }^{5}$ (fig. $\mathbf{n}^{\circ}$ 1). Puis, en 1984, étaient publiées, coup sur coup, deux synthèses, qui font toujours référence : Le Site balnéaire par Dominique Rouillard, et le catalogue de l'exposition Les villes d'eaux en France (ouverte en janvier 1985), organisée par l'Institut français d'architecture, sous la direction de Lise Grenier'. Le mouvement était lancé.

\section{L'exploration méthodique du rivage : quinze ans d'études à I'Inventaire général}


Figure 2

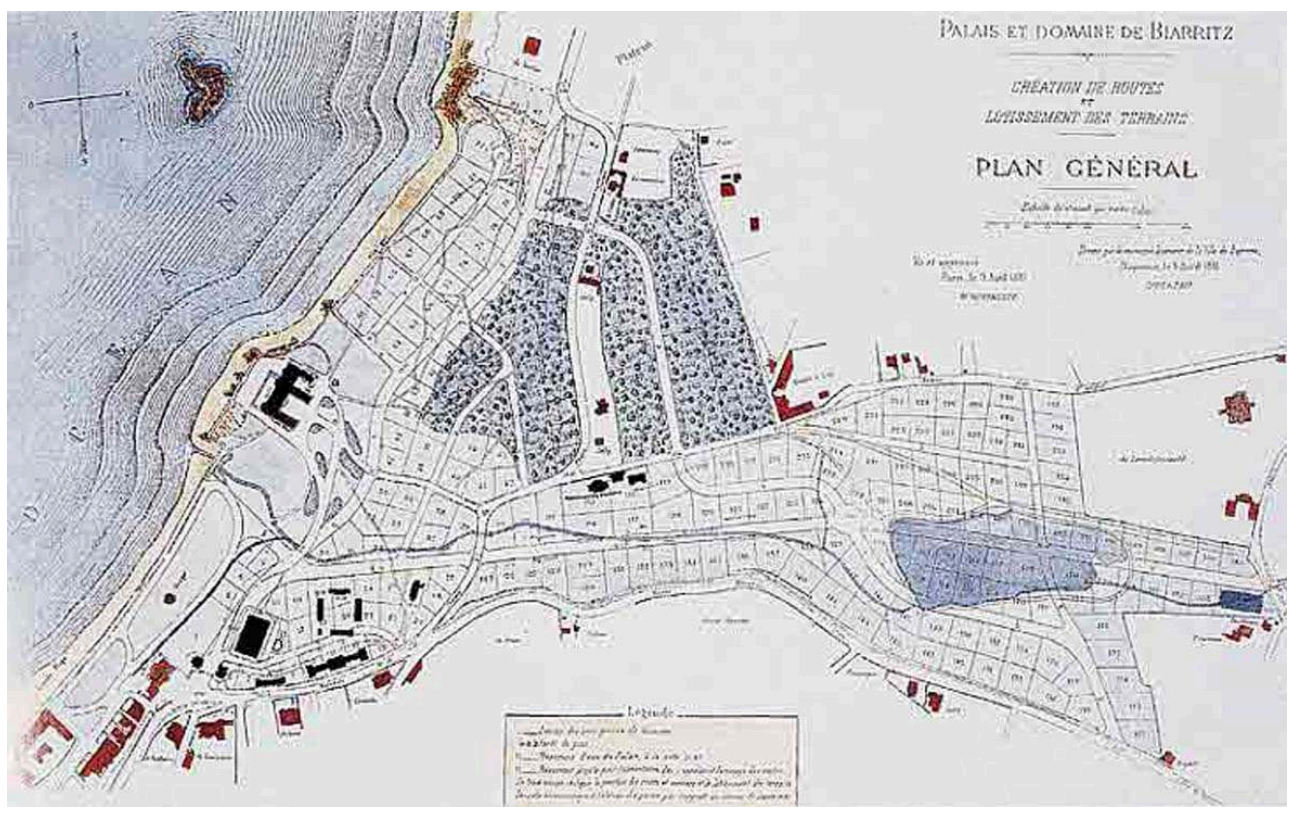

Biarritz (Pyrénées-Atlantiques). Plan du lotissement des terrains du domaine impérial, 1881. Repro. Coll. IFA. Extrait : VILles D'EAUX. Paris, Dexia/Imprimerie Nationale, 2002, p. 29.

5 Tandis que l'I.F.A. poursuivait la réévaluation sensible des grandes stations (fig. $\left.\mathbf{n}^{0} \mathbf{2}\right)^{7}$, l'Inventaire général à son tour s'engageait progressivement dans une exploration méthodique du rivage. En 1988 déjà, Françoise Hamon avait organisé un premier stage de l'Ecole du patrimoine sur le thème ${ }^{8}$, tandis que Claude Laroche travaillait à une étude approfondie de la station d'Hossegor'. En 1989, sous mon impulsion, le «Laboratoire de recherches sur le patrimoine français» (UMR 22) affichait l'architecture de villégiature comme thème prioritaire dans le champ de l'architecture ${ }^{10}$. 
Figure 3

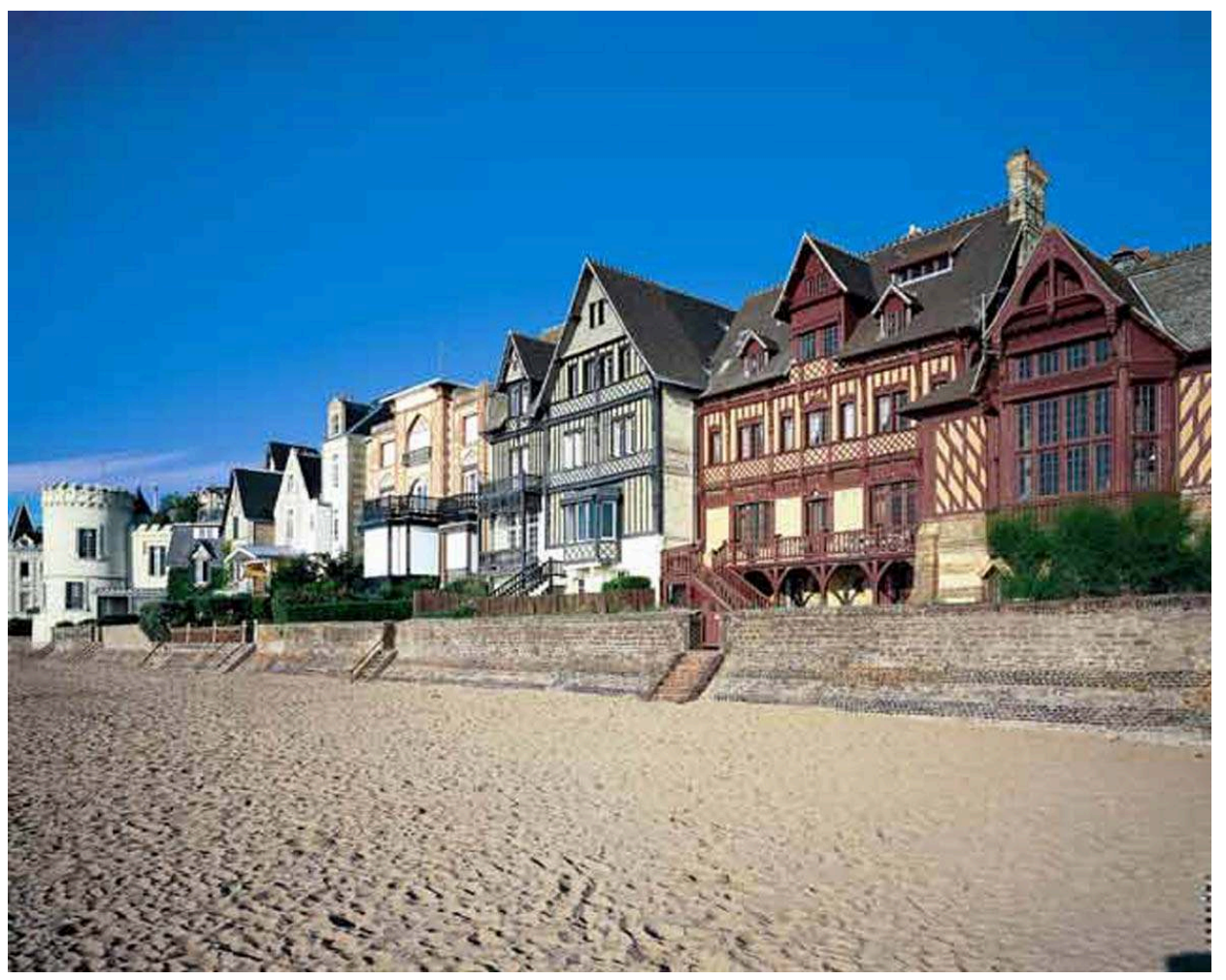

Trouville-sur-Mer (Calvados). Digue et front de mer. Phot. C. Rose. Extrait : villes d'EAux. Paris, Dexia/ Imprimerie Nationale, 2002, p. 38.

6 L'étude sur Trouville avait montré la fécondité du dépouillement systématique des guides touristiques des stations, comme d'autres le montraient ailleurs ${ }^{11}\left(\right.$ fig. $\mathbf{n}^{\circ} \mathbf{3}$ ). S'appuyant sur des dépouillements antérieurs de recueils et de revues, étaient établies les premières listes d'images d'édifices balnéaires, classées par stations, et engagées les premières études sur les stations de la côte normande ${ }^{12}$. Ces rapports internes servirent de base à la publication des premières plaquettes, éditées dans la collection des " Itinéraires du Patrimoine ", visant à sensibiliser le public à cette nouvelle dimension patrimoniale ${ }^{13}$.

7 En 1993, Bernard Toulier établissait pour la Revue de l'art ${ }^{14}$ un premier bilan des recherches conduites par le service de l'Inventaire, comme par ses partenaires, IFA, Ecoles d'architecture, bref par ce qui commençait à constituer un vrai réseau de recherche sur le phénomène de la Villégiature. Les principaux acteurs de ce réseau se retrouvaient à Biarritz, pour des journées d'études sur «l'Architecture balnéaire du littoral », en septembre 1993, clôturant ainsi l'opération «Mon patrimoine » mise en œuvre durant l'été sur la côte basque.

8 Cette année-là, selon un heureux hasard objectif, Alain Charles publiait son étude suggestive sur les Chalets et villas de La Baule ${ }^{15}$, la station où neuf ans plus tard, en ce mois d'octobre 2002, nous sommes réunis, pour la sixième fois, après les rencontres successives de Biarritz (23-25 septembre 1993), Cannes (20-22 avril 1994), Dinard (1995), Deauville (23-25 octobre 1997), Hyères (1999), pour faire un nouveau point sur «les Réseaux de la Villégiature ». 
Figure 4

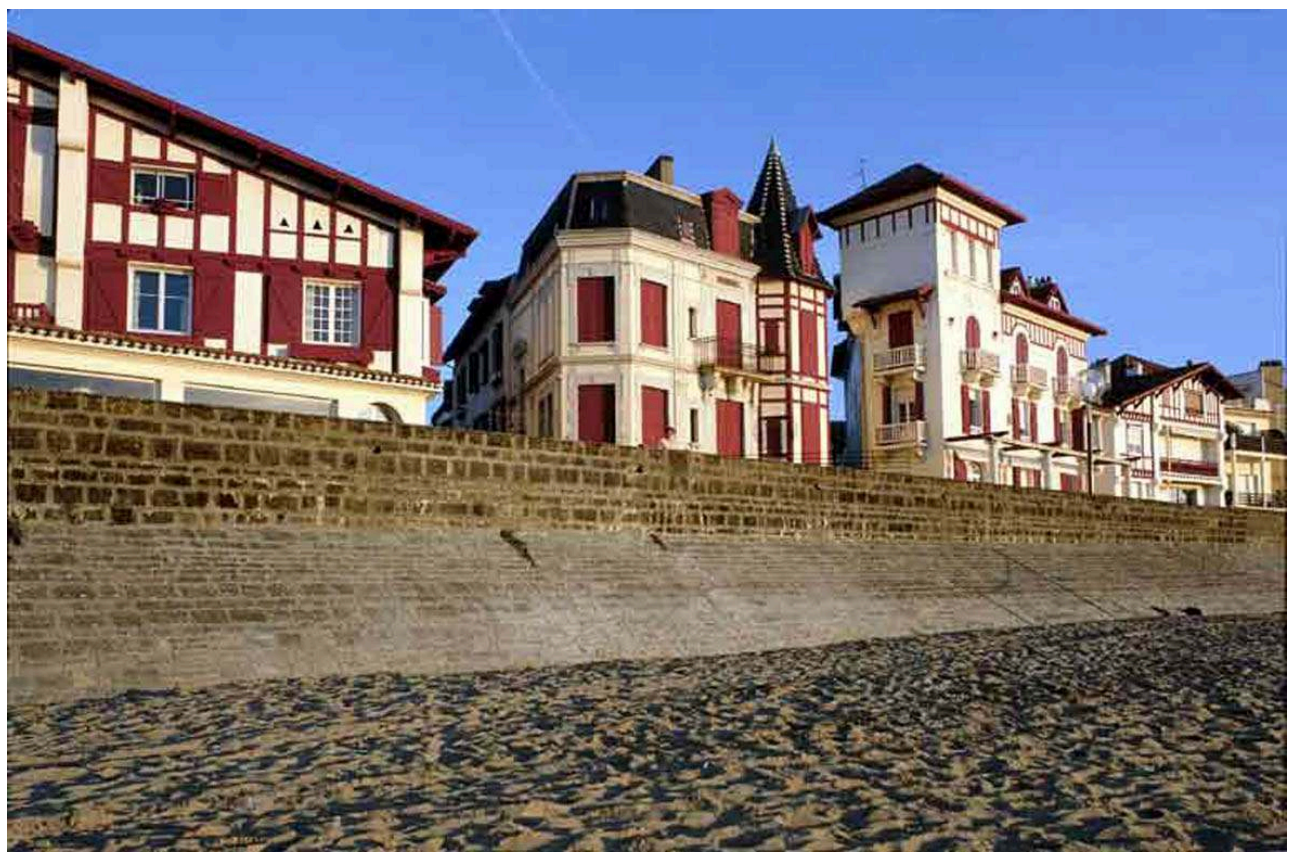

Saint-Jean-de-Luz (Pyrénées-Atlantiques). Digue et front de mer. Phot. C. Rose. Extrait : ViLLEs d’EAUX. Paris, Dexia/Imprimerie Nationale, 2002, p. 39.

9 Tandis que se poursuivaient les recherches autour des deux premiers pôles, la côte normande ${ }^{16}$ et la côte basque ${ }^{17}$, (fig. $\mathbf{n}^{\circ}$ 4) des études conduites par des architectes, des étudiants, jeunes doctorants et chercheurs indépendants, liés plus ou moins directement à l'UMR ou aux 22 services régionaux de l'Inventaire, souvent en partenariat avec l'IFA ou les Ecoles doctorales des universités, se mettaient en place pour conduire aux premières publications sur la côte d'Opale ${ }^{18}$, la côte d'Emeraude ${ }^{19}$, la côte de Granit rose ${ }^{20}$, Royann ${ }^{21}$, la Côte d'Azurr22. Peu à peu, ces monographies de stations ou de "Côtes", formaient une chaîne presque continue sur le littoral, qui permet d'établir une bibliographie géographique ${ }^{23}$, en attendant l'établissement d'un véritable atlas historique, plusieurs fois annoncé, mais toujours en chantier.

\section{De la villa balnéaire à la ville de villégiature}


Figure 5

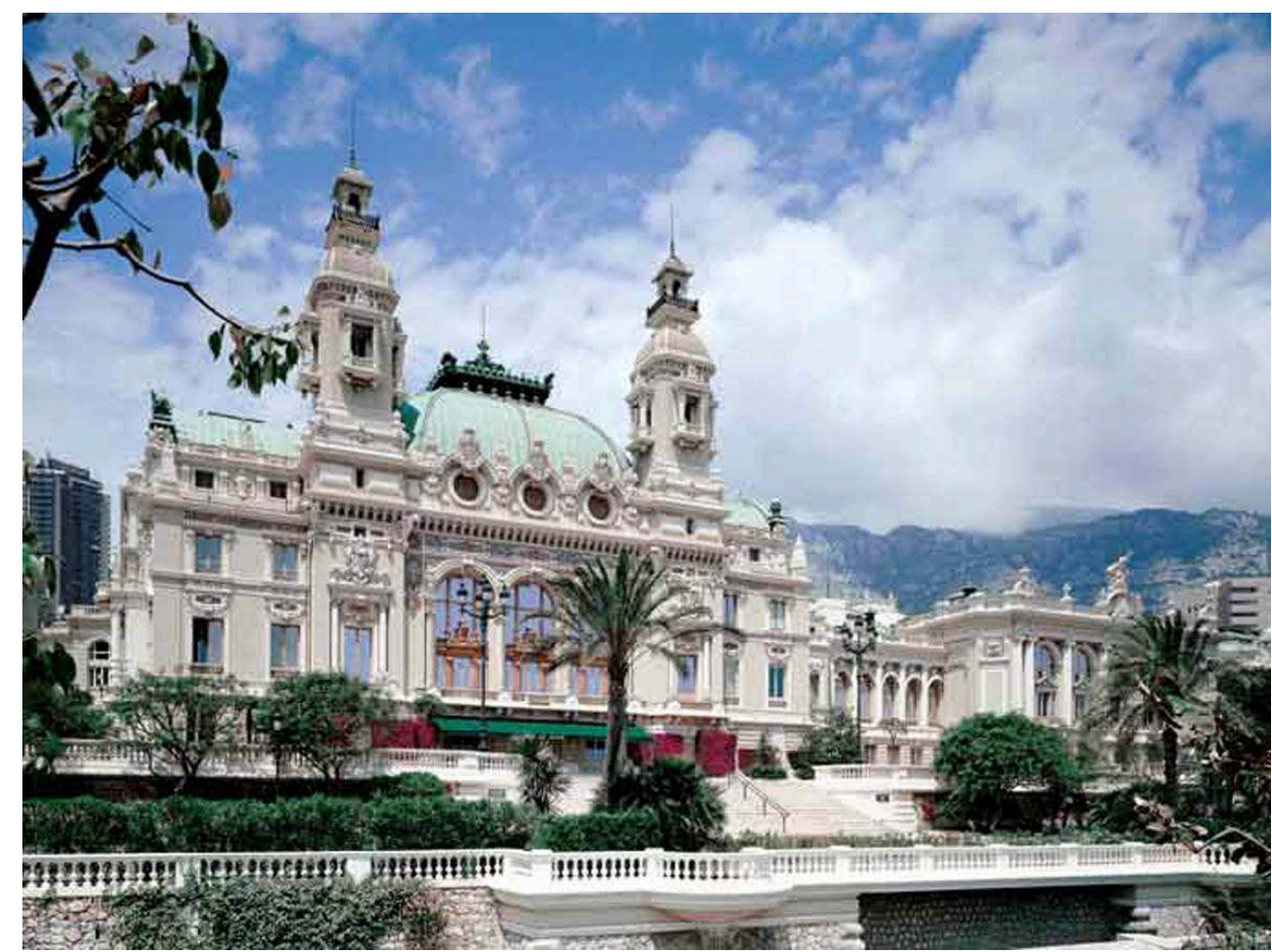

Monte-Carlo (Principauté de Monaco). Casino, façade méridionale. Phot. C. Rose. Extrait : viLLes D'EAUX. Paris, Dexia/Imprimerie Nationale, 2002, p. 92-94.

10 La découverte de l'importance du thème de la villégiature dans l'histoire de l'architecture contemporaine s'est faite, semble-t-il, concentriquement (fig. $\mathbf{n}^{\circ} \mathbf{5}$ ). 
Figure 6

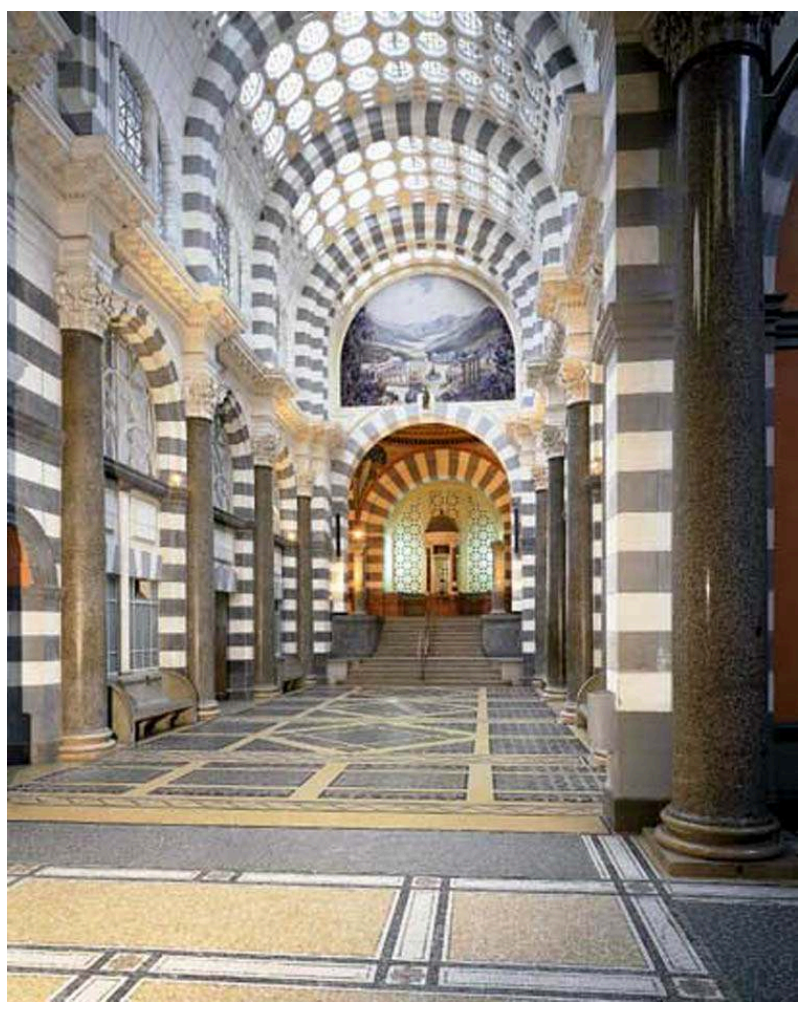

Le Mont-Dore (Puy-de-Dôme). Etablissement thermal, galerie centrale. Phot. C. Rose. Extrait : Villes d'eaux. Paris, Dexia/Imprimerie Nationale, 2002, p. 61-62.

11 On fut d'abord sensible à la villa pittoresque, déclinant toutes les gammes du pittoresque ${ }^{24}$, puis aux grands équipements, casinos $^{25}$, grands hôtels ${ }^{26}$, établissements thermaux ${ }^{27}$, (fig. $\mathbf{n}^{\circ} \mathbf{6}$ ) établissements de bains de mer (fig. $\mathbf{n}^{\circ} \mathbf{7}^{28}$.

On aborda le phénomène par type de station, thermale, balnéaire, avant d'intégrer aussi les villégiatures "de bord de ville», selon l'expression heureuse avancée par François Loyer ${ }^{29}$, et plus récemment de $"$ bord de fleuve $»^{30}$. 


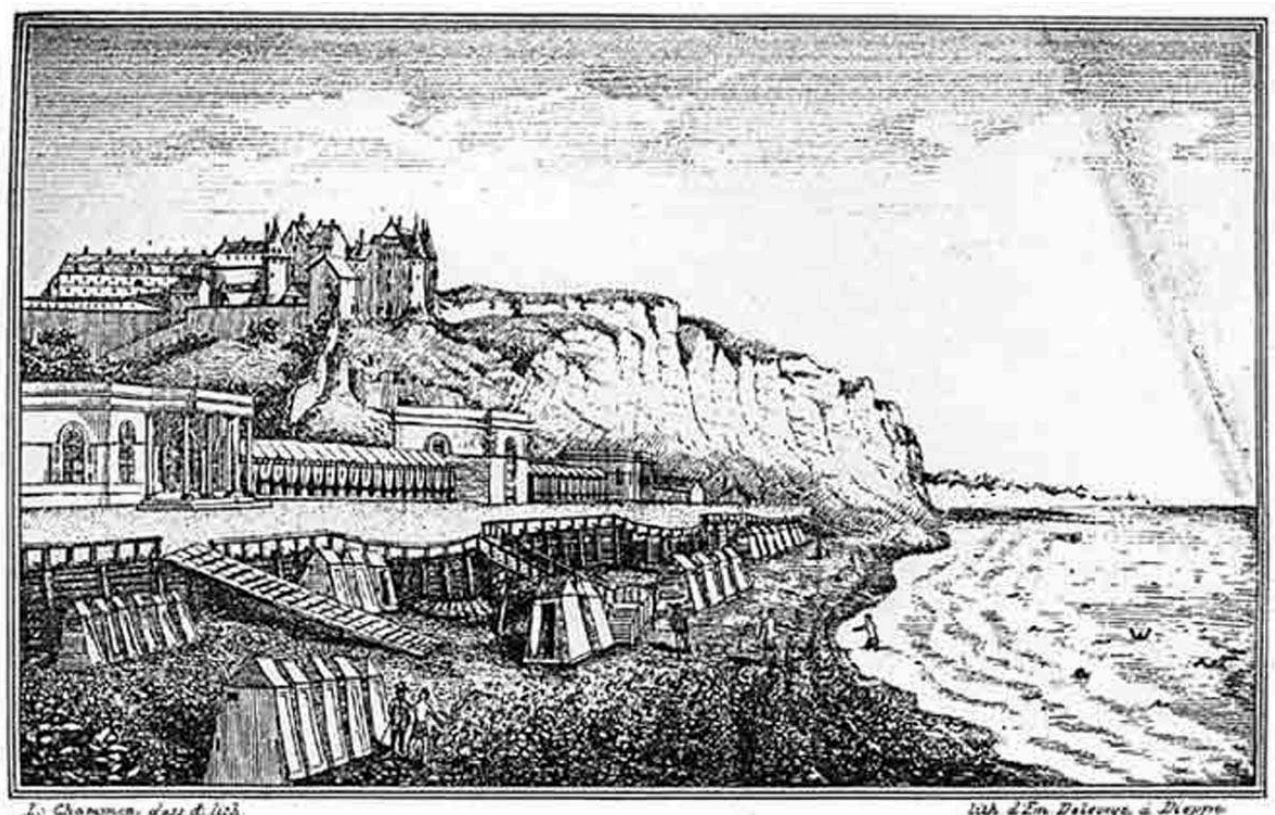

Dieppe (Seine-Maritime). Etablissement de bains chauds et de bains froids. Gravure, 1885. Coll. B. Toulier. Extrait : VILLES D'EAUX. Paris, Dexia/Imprimerie Nationale, 2002, p. 88.

13 La prise de conscience de la dimension urbaine globale de ce qui paraissait d'abord réglé par le caprice, l'initiative individuelle et le plaisir fut plus tardive. On mesure mieux aujourd'hui à quel point ces villes neuves furent un laboratoire d'urbanisme décisif : comme après l'école de Barbizon, impressionnisme, post-impressionnisme et fauvisme se construisent face à l'art pompier, et cela en plein air - souvent l'air du large -, villes d'eau, villes balnéaires et même villes de villégiature « de bord de ville » sont la stricte contrepartie de l'urbanisme haussmannien, où triomphe le style "Beaux-Arts ", mais aussi le contre-pied et souvent le complément de la sociabilité aristocratique estivale de la villégiature de château.

14 On mesure mieux aussi à quel point le programme - cure de santé et de plaisir, station mondaine ou familiale, quartier de villégiature s'accrochant à un port ancien ou ville neuve construite sur la dune ou la falaise - est plus déterminant que les styles, qui habillent les villas, comme les modes colorent les tenues des élégantes estivantes.

Les premières études synthétiques furent sémiologiques - le discours sur le site balnéaire construisant un nouvel imaginaire du rivage -, monographiques - études de stations, études des éléments majeurs: casinos, grands hôtels, établissements hydrothérapiques - , thématiques - villes d'eaux, stations d'hiver, stations d'été, villégiatures de bord de mer, de fleuve et de ville. Nous mesurons mieux aujourd'hui la convergence de ces réseaux qui se construisent sur le maillage des voies ferrées, puis sur celui du nouveau réseau automobile ou aéronautique, mais aussi la multiplicité des échelles : Paris rayonnant vers toutes les plages - gare du Nord, Le Touquet-Paris-Plage, gare Saint-Lazare, Trouville-Deauville, gare Montparnasse, Dinard, La Baule, etc.; capitales régionales et leurs rivages proches : de la bourgeoisie de Caen et du Havre villégiaturant sur les plages normandes, déjà au temps de l'adolescence de Flaubert, aux industriels du Nord bâtissant sur la côte d'Opale. 

même aspiration à échapper à la ville contemporaine ${ }^{31}$. Mais ce nouvel espace qui se construit sur le vide, le long du rivage ou autour de l'eau courante ou cristallisée, est aussi traversé de tensions constitutives : viaire cohérent, linéaire ou rayonnant, et variété étourdissante des styles de maisons, beaucoup plus ouverte qu'en ville ;

centrifuges, ou l'on cherche loin de la ville un dialogue plus direct avec la nature, même si on se plaît à le filtrer par différents dispositifs - bow-windows, vérandas, larges baies - et forces centripètes d'une nouvelle sociabilité de villes de villégiature, construite autour des nouveaux monuments du loisir - casinos (fig. $\mathbf{n}^{\circ} \mathbf{9}$ ), palaces, promenades de bord de mer, avenues plantées ou simples planches, etc.;

récurrence de ces éléments constitutifs, mais aussi variété des formes urbaines selon la nature de la relation à l'eau, comme l'avait déjà bien souligné Lise Grenier en 1984 : point d'émergence des stations thermales, front de mer, bord de fleuve ou de lac (fig. $n^{\circ}$ 10).

Figure 8

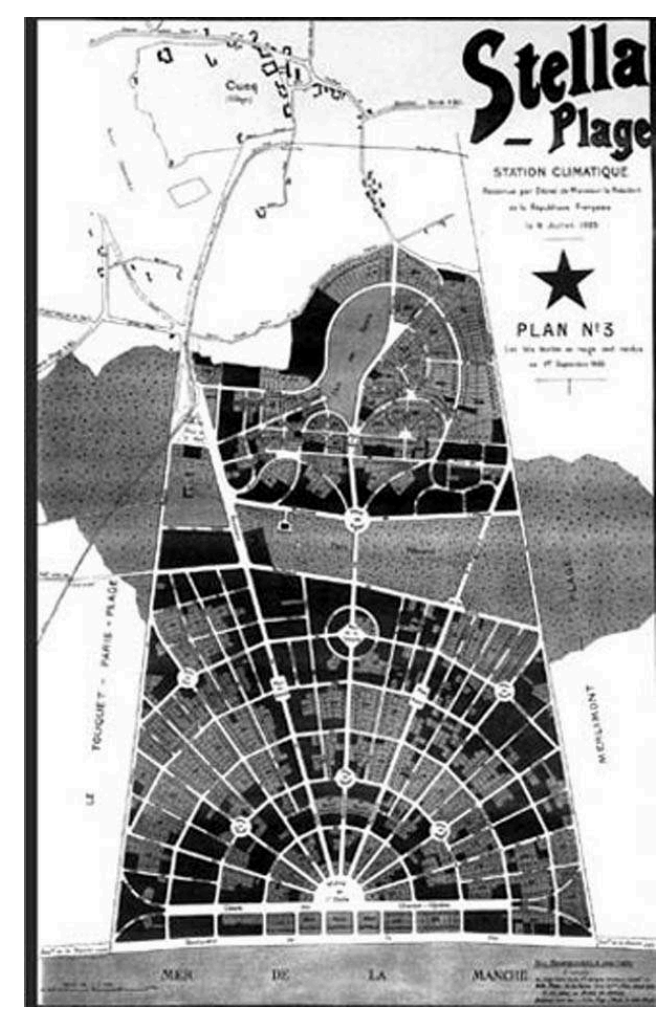

Stella-Plage (Pas-de-Calais). Plan du lotissement, 1925. Repro., doc. IFA. Extrait : VILLES D'EAUX. Paris, Dexia/Imprimerie Nationale, 2002, p. 32. 
Figure 9

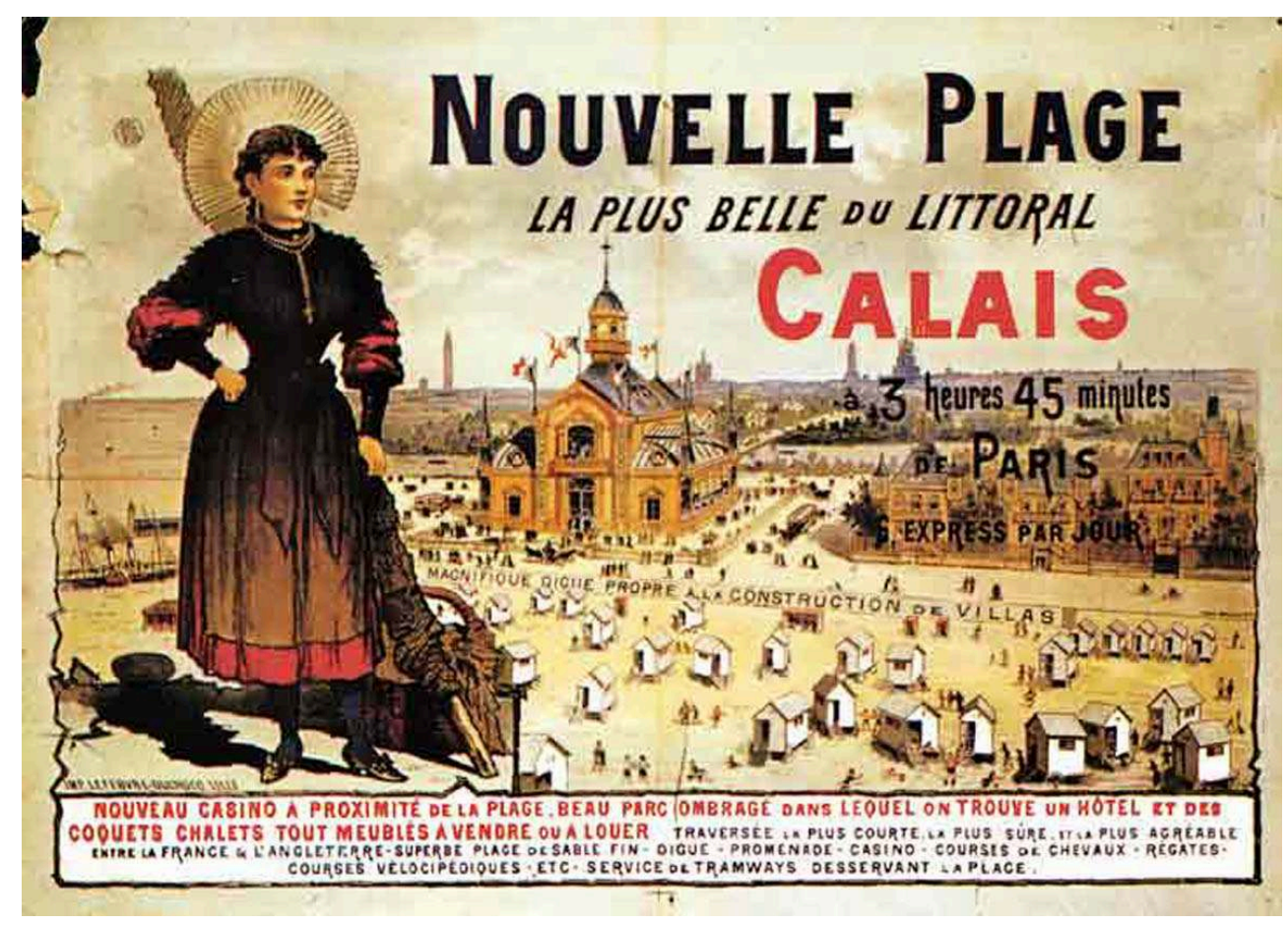

Calais (Pas-de-Calais). Nouvelle plage construite autour du casino. Affiche, s.d. (fin XIXe s. ?). Repro. Coll. B. Toulier. Extrait : VILLES D'EAUX. Paris, Dexia/Imprimerie Nationale, 2002, p. 30.

Figure 10

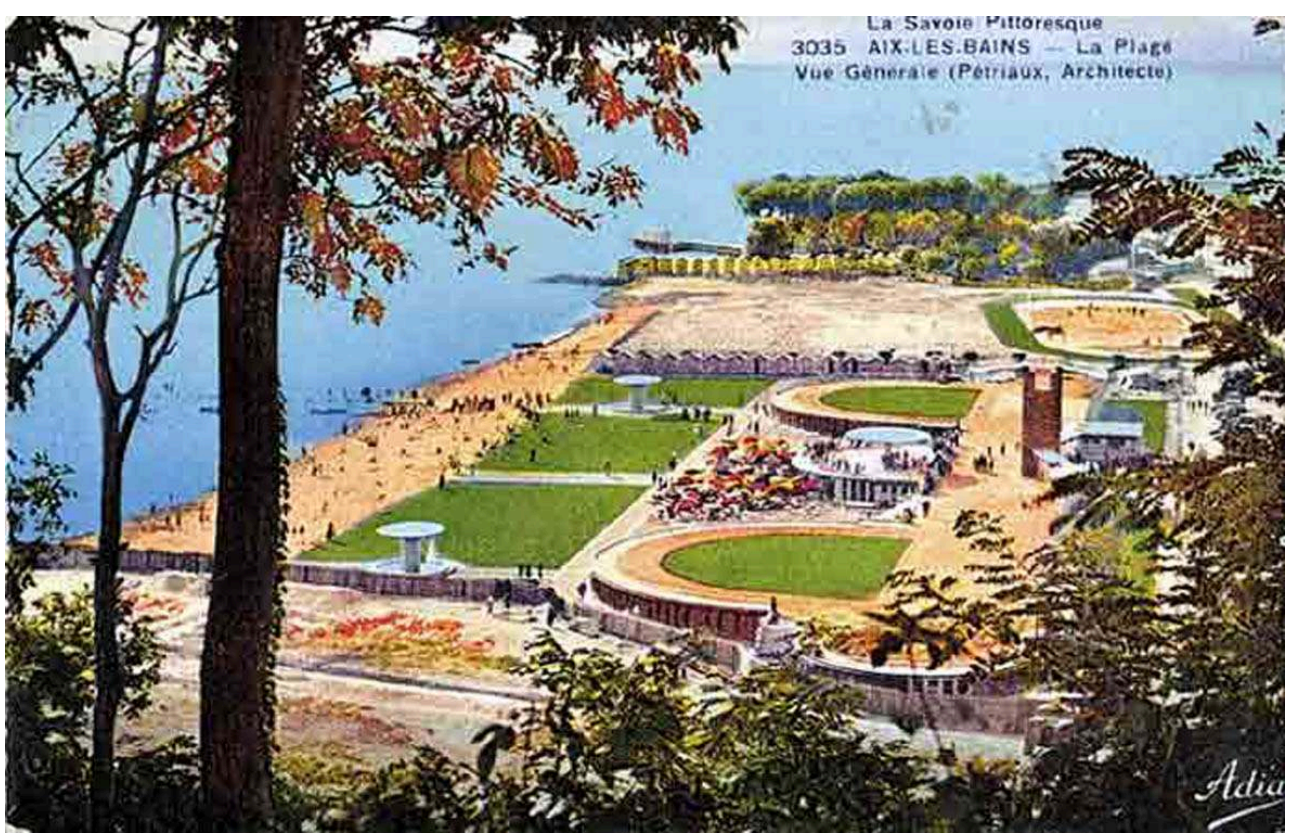

Aix-les-Bains (Savoie). Nouvelle plage au bord du lac du Bourget. Carte postale, s.d. (années trente) Coll. B. Toulier. Extrait : VILLES D'EAUX. Paris, Dexia/Imprimerie Nationale, 2002, p. 114.

En 1953, dans Les vacances de M. Hulot, Jacques Tati célébrait la villégiature des congés payés de l'après-guerre ; en 1981, Lola de Jacques Demy s'ouvrait sur le panoramique de la grande plage de La Baule, où roule la Cadillac blanche de l'amour perdu de l'héroïne : pour le cinéma, l'espace de villégiature était un espace social, comique ou mythique. 
Dans les années suivantes, le rivage devenait un espace plus architectural : Eric Rohmer filmait successivement les côtes de la Manche dans Pauline à la plage, Biarritz et SaintJean-de-Luz dans Le Rayon vert, Dinard dans Conte d'été. Tandis que l'utopie urbaine reste à réinventer (après Tati établissant dans Play time le constat humoristique de la faillite de l'urbanisme parisien des Trente glorieuses, Cédric Klapisch filme la capitale envahie par les sables (Peut-être), science-fictionnant par anticipation le Paris-plage de l'été 2002), nous prenons mieux la mesure de la profondeur historique de ce nouveau territoire du plein saisonnier et la complexité des "réseaux de la Villégiature ", qui nous occupent ici.

\section{NOTES}

1. FOUCART, Bruno. Monuments historiques. 1974.

2. MIGNOT, Claude. L'architecture au XIX e siècle. Fribourg: Office du livre / Paris: Le Moniteur, 1983 (co-éd. allem., 1983 ; co-éd. angl., New York, Rizzoli, 1983) ; LOYER, François. Le siècle de l'industrie, 1789-1914. Paris/Lausanne : Skira, 1983.

3. CORBIN, Alain. Le territoire du vide, l'Occident et le désir de rivage, 1750-1840. Paris : Aubier, 1988.

4. Voir le numéro spécial de la revue Monuments historiques, "Retour aux sources", notamment FOUCART, Bruno. Au plaisir des architectes. Les villes d'eau et leur architecture aux $\mathrm{XIX}^{\mathrm{e}}$ et $\mathrm{XX}^{\mathrm{e}}$ siècles. Monuments historiques, 1978, $\mathrm{n}^{\circ}$ 1. P. 2-11. Pour une autre étude pionnière, HAMON, Françoise. L'architecture balnéaire de la côte d'Emeraude. Mémoires de la société d'histoire et d'archéologie de Bretagne, 1981, t. LVIII. P. 5-21.

5. CONTAL, Marie-Hélène. Vittel 1854-1936 : création d'une ville thermale. Le Moniteur, 1982 ; CULOT, Maurice (dir.). La ville d'hiver d'Arcachon. Liège : Mardaga, 1983 (rééd. sous le titre : Arcachon, la ville d'hiver. Liège : Mardaga, 1988).

6. ROUILLARD, Dominique. Le site balnéaire. Liège : Mardaga, 1984 ; GRENIER, Lise (dir.). Les villes d'eaux en France. Paris : IFA, 1984.

7. CULOT, Maurice, JAKAVLJEVIC, Nada (dir.). Trouville. Liège : Mardaga, 1989 ; CULOT, Maurice, MESURET, Geneviève (dir.). Architectures de Biarritz et de la côte Basque : de la Belle Epoque aux années trente. Liège: Mardaga, 1990 ; CULOT, Maurice, PAVLOVSKY, Jacques. La Côte Basque des années trente: architectures d'André Pavlovsky. Paris: Norma, 1991 ; CULOT, Maurice (dir.). La côte normande des années trente : Trouville-Deauville, société et architecture balnéaires 1910-1940. Paris: Norma,1992; CULOT, Maurice, RIBETON, Olivier, MESURET, Geneviève et al.. Biarritz. Villas et jardins. 1900-1930. Paris : Norma, 1992. Pour la suite des publications patronnées par l'I.F.A. ou publiées en partenariat avec lui, voir infra notes XV et XVI.

8. Elle avait publié sept ans plus tôt un travail précurseur, voir ci-dessus, note IV.

9. LAROCHE, Claude. La côte landaise des années trente : Hossegor 1920-1940 : la station des sports élégants. Paris : Norma, 1991; ID. Hossegor : 1923-1939: architecture et identité régionale. Bordeaux : APIA ; le Festin, 1993 (coll. Cahiers du patrimoine, $n^{\circ} 32$ ).

10. Voir le site

11. BIHR, Jean-Pierre. Regards sur la Côte d'Emeraude. Guides touristiques 1883-1939. SaintJacut-de-la-Mer, 1991. P. 5. 
12. Thierry KOZAK et Gilles PLUM rendirent plusieurs rapports successifs, qui formèrent le premier fonds documentaire interne au laboratoire de l'Inventaire, noyau autour duquel se forma une documentation informelle de sources, de travaux inédits et de publications.

13. LIBOUREL, Jean-Louis. Villas de Houlgate : Calvados. [Caen] : Développement culturel en Basse-Normandie, 1991 (coll. Itinéraires du patrimoine, n³); D’ABOVILLE, Christine, COLONNIER, Maguelonne, ETIENNE-STEINER, Claire. Sainte-Adresse et le Nice havrais, SeineMaritime. [Rouen] : Connaissance du patrimoine de Haute-Normandie, 1992 (coll. Itinéraires du patrimoine, $\mathrm{n}^{\circ} 8$ ) ; PLUM, Gilles. Villas d'Arromanches-les-Bains : Asnelles, Tracy-sur-Mer, Ver-sur-Mer. [Caen] : Développement culturel en Basse-Normandie, 1993 (coll. Itinéraires du patrimoine, $n^{\circ} 42$ ). Pour la suite voir infra note XIV.

14. TOULIER, Bernard. L'architecture des bains de mer: un patrimoine marginalisé. Revue de l'art, 1993, n 101. P. 29-40.

15. CHARLES, Alain. La Baule et ses villas. Paris : Ed. Massin, 2002.

16. Voir, après les premières études citées supra note XII, LECHERBONNIER, Yannick, PELVILLAIN, Hervé. Villas de Lions-sur-Mer et Hermanville-sur-Mer : Calvados. [Caen]: Développement culturel en Basse-Normandie, 1996. (coll. Itinéraires du patrimoine, $\mathrm{n}^{\circ} 125$ ); ETIENNE-STEINER, Claire. Le Havre : ville, port et agglomération. [Rouen] : Connaissance du patrimoine de Haute-Normandie, 1999 (coll. Indicateurs du patrimoine, $n^{\circ} 11$ ) ; TOURNOUX, Marie-Noëlle. Deauville, les styles normands. [Caen]: Développement culturel en BasseNormandie, 1999 (coll. Itinéraires du patrimoine, $n^{\circ} 212$ ). Ainsi que des travaux inédits : TISSOT, Claire. Luc-sur-mer, Station balnéaire des familles. Mémoire de maîtrise, Université de Tours, 1997 ; DUJARDIN, Bénédicte. Saint-Valéry en Caux. DEA, Université de Paris IV, 1999.

17. Voir, après les premières études citées supra note VII, CULOT, Maurice, TOULIER, Bernard (dir). Le Pays basque. Architectures des années 20 et 30. Paris : Norma, 1993; et la grande enquête de Claude LAROCHE sur la côte basque (en cours).

18. KLEIN, Richard. Le Touquet Paris-Plage : la côte d'Opale des années trente. Paris : Norma, 1994 ; KLEIN, Richard (dir.). La Côte d'Opale : architectures des années 20 et 30 : Wissant, Ambleteuse, Wimereux, Hardelot, Le Touquet, Stella-Plage, Merdimont, Berck. Paris : Norma, 1998. Voir aussi, CORBEL, Pierre-Yves. Malo-les-Bains... Dunkerque : dunes, briques et béton. Lille : Association Christophe Dieudonné, 1995 (coll. Cahiers du patrimoine, $n^{\circ} 41$ ).

19. DELIGNON, Gaëlle. Saint-Malo-Paramé. Urbanisme et architecture balnéaires. 1840-1940. Rennes : PUF, 1999 ; MUEL, Francis, TOULIER, Bernard, dir. La côte d'Emeraude. Paris : Monum/ Ed. du patrimoine, 2001. Ces ouvrages montrent implicitement les limites des études antérieures : Modernité et régionalisme, Bretagne 1918-1945. Liège: Pierre Mardaga éditeur, 1986. LE COUEDIC, Daniel. Les architectes et l'idée bretonne, 1904-1945. Rennes : SHAB-AMAB, 1995, [voir notamment le chapitre sur Le laboratoire balnéaire, p. 308-331] ; LOYER, François, GUENE, Hélène. L'Eglise, l'Etat et les architectes, Rennes, 1870-1940. Paris : Norma, 1995.

20. JUSTOME, Elisabeth. Urbanisme et architecture balnéaire sur la côte de Granit rose. DEA, Université de Rennes II, 1999.

21. Royan années 50. Parcours d'architecture du XXe siècle. Royan : CAUE, 2000.

22. STEVE, Michel. La métaphore méditerranéenne. L'architecture sur la Riviera de 1860 à 1914. Nice: Demaistre, 1996. Voir aussi STEVE, Michel. L'architecture Belle Epoque à Nice. Nice : Demaistre, 1995 (coll. Guides d'Azur, $n^{\circ}$ 1) et ID. La Riviera de Charles Garnier. Nice : Demaistre, 1998 (coll. Guides d'Azur, n 3) ; ESCRIBE, Dominique. La Côte d'Azur, genèse d'un mythe. Nice: ACAM, 1998 ; BILAS, Charles, ROSSO, Lucien. La Côte d'Azur, années 20 et 30. Paris : Telleri, 1999. Voir aussi les études de stations particulières : MILLIET-MONDON, Camille. Cannes, 1835-1914. Villégiature, urbanisation, architecture. Nice : Serre, 1986 ; ID. Cannes, architectures de villégiature $\mathbf{n}^{\circ} 1$. Aix-en-Provence: Association pour le patrimoine de Provence, 1994 (coll. Itinéraires du patrimoine, $n^{\circ} 49$ ) ; NEGREL, Geneviève. Hyères, la ville climatique. Aix-en-Provence : Association pour le patrimoine de Provence, 1996. (coll. Itinéraires 
du patrimoine, $\mathrm{n}^{\circ}$ 126) ; FRAY, François, NEGREL, Geneviève. Hyères, Var. Aix-en-Provence : Association pour le patrimoine de Provence, 2000 (coll. Images du patrimoine, $\mathrm{n}^{\circ} 198$ ); BERTRAND, Nathalie. Tamaris, villégiature, urbanisme, architecture dans la seconde moitié du XIX e siècle. Thèse de doctorat, Université d'Aix-Marseille, 2000.

23. Voir la bibliographie compilée, sous la direction de Bernard TOULIER, par Geneviève-Marie MARION. A paraître sur le site Internet Puis faire Projets et recherche, Programme du CNRS, UMR 22 sur l'architecture de la villégiature.

24. PEROUSE DE MONTCLOS, Jean-Marie. De la villa rustique au pavillon de Banlieue. Revue de l'art, 1976, n 32. P. 23-36; ID. Le chalet à la suisse, fortune d'un modèle vernaculaire. Architectura, 1987. P. 76-95 ; Le régionalisme. $\mathrm{N}^{\circ}$ spécial de Monuments historiques, $\mathrm{n}^{\circ} 189$, 1993.

25. CHABILAND, Nathalie. Les casinos, définition d'un type d'architecture balnéaire. Mémoire de maîtrise. Paris : Université de Paris IV, 1992 ; TOULIER, Bernard, GUILLEMAIN, Hervé, HARTELASSERRE, Pierre Jean, et al. Biarritz, Le casino.1920-1994. Paris : Norma, 1994.

26. Après un livre qui aborde le thème sous son plus grand angle (WATKIN, David, BOUVET, Vincent et al. Palaces et grands hôtels d'Europe. Paris : Flammarion, 1984), voir THIEBAUD, Eric. Les palaces des stations balnéaires à la Belle Epoque. Mémoire de maîtrise. Paris : Université de Paris IV, 1995 ; KOSSINKI, Sophie, MICHELETTI, Eric. Grands hôtels du bord de mer. Paris : Histoire et collections, 1996.

27. Sur les thermes, l'exposition pionnière organisée en 1985 par Lise Grenier n'avait pas permis de publier toute la documentation exploitée. Les études sur le sujet ont été poursuivies par un des membres de l'équipe, sans infléchir fondamentalement les lignes qui avaient été fermement tracées en 1985 par L. Grenier: JARASSE, Dominique. Les thermes romantiques. Bains et villégiature en France de 1800 à 1850. Clermont-Ferrand : Institut d'études du Massif central, 1992. Voir aussi dans la même collection «Thermalisme et civilisations » publiée par le Centre d'études sur le thermalisme de l'université Blaise-Pascal (Clermont II): Villes d'eaux des Pyrénées occidentales. Patrimoine et devenir. Clermont-Ferrand, 1996. Les services régionaux de l'Inventaire publièrent des études monographiques: LEONE-ROBI, Isabelle. Bagnoles-del'Orne, ville d'eaux. [Caen] : Développement culturel en Basse-Normandie, 1995. (coll. Itinéraires $\mathrm{du}$ patrimoine, $\mathrm{n}^{\circ}$ 106) ; CERONI, Brigitte, FIZELLIER-SAUGET, Bernadette, LAFONT, Annie. Le Mont-Dore: une ville d'eaux en Auvergne, Puy-de-Dôme. Clermont-Ferrand: Etude du patrimoine auvergnat, 1998 (coll. Images du Patrimoine, $n^{\circ}$ 175) ; La Bourboule [à paraître].

28. CHABILAND, Nathalie. Les établissements de bains de mer. DEA. Paris : université de Paris IV, 1993.

29. CUEILLE, Sophie. Le Vésinet, modèle français d'urbanisme paysager. Paris : APPIF, 1989 (coll. Cahier de l'Inventaire, $\mathrm{n}^{\circ} 17$ ) (rééd., Cahier du patrimoine, 1999); CUEILLE, Sophie. Maisons-Laffitte, parc, paysage et villégiature, 1630-1930. Paris : APPIF, 1999 (coll. Cahier du patrimoine, $\left.\mathrm{n}^{\circ} 53\right)$.

30. HERVIER, Dominique (dir.), BUSSIERE, Roselyne (réd.). Saint-Germain-en-Laye, 1800-1940 : Yvelines. Paris : APPIF, 1997 ; BUSSIERE, Roselyne, et al. Au sud de Versailles. Buc, Jouy-enJosas, Les Loges-en-Josas, Toussus-Le-Noble. Yvelines. Paris : APPIF, 2001.

31. TOULIER, Bernard. Villes d'eaux, stations thermales et balnéaires. Paris: Imprimerie nationale/ Dexia éditions, 2002. 


\section{INDEX}

Mots-clés : villégiature, réseaux, Bruno Foucart, François Loyer, casino, Grand hôtel, châteaux, hôtels, habitat ouvrier, villas de bord de mer, gratte, ciel, villes de villégiature, Maurice Culot, Vittel, Arcachon, Dominique Rouillard, villes d'eaux, Lise Grenier, Françoise Hamon, Claude Laroche, Hossegor, Trouville, Bernard Toulier, Alain Charles, La Baule, côte normande, côte basque, Côte d'Opale, Côte d'Emeraude, Côte de Granit rose, Royan, Côte d'Azur, établissements thermaux, établissements de bains de mer, station thermale, station balnéaire, Le Touquet, Paris, Plage, Trouville, Deauville, Dinard, bow, window, véranda, palaces, promenades de bord de mer, avenues, front de mer, Jacques Tati, Eric Rohmer, côtes de la Manche, Biarritz, Dinard, Cédric Klapisch

\section{AUTEUR}

\section{CLAUDE MIGNOT}

Professeur à l'université de Paris IV, directeur de la FRE 2514 (ancienne UMR 22)

ClaudeN.Mignot@wanadoo.fr 